\title{
Dislocation of Rab13 and vasodilator-stimulated phosphoprotein in inactive colon epithelium in patients with Crohn's disease
}

\author{
MIZUKI OHIRA $^{1}$, NOBUHIDE OSHITANI ${ }^{1}$, SHUHEI HOSOMI ${ }^{1}$, \\ KENJI WATANABE ${ }^{1}$, HIROKAZU YAMAGAMI $^{1}$, KAZUNARI TOMINAGA ${ }^{1}$, \\ TOSHIO WATANABE ${ }^{1}$, YASUHIRO FUJIWARA ${ }^{1}$, KIYOSHI MAEDA ${ }^{2}$, \\ KOSEI HIRAKAWA ${ }^{2}$ and TETSUO ARAKAWA ${ }^{1}$ \\ Departments of ${ }^{1}$ Gastroenterology; ${ }^{2}$ Surgery, Osaka City University \\ Graduate School of Medicine, Osaka, Japan
}

Received May 5, 2009; Accepted July 3, 2009

DOI: $10.3892 /$ ijmm_00000300

\begin{abstract}
Crohn's disease is associated with increased permeability of the intestine even in quiescent patients. Increased intestinal permeability may cause dysregulated immunological responses in the intestinal mucosa that leads to chronic intestinal inflammation. Tight junction proteins contribute to intestinal permeability, and functional abnormality and dislocation of such proteins may cause increased intestinal permeability. We studied the expression of tight junction proteins Rab13, vasodilator-stimulated phosphoprotein (VASP), zonula occludin-1 (ZO-1), and F-actin in the intestinal epithelium of patients with inactive inflammatory bowel disease. Surgical samples were obtained from 10 controls (without inflammatory bowel disease), 10 patients with Crohn's disease and 7 patients with ulcerative colitis. F-actin was visualized with fluorescent phalloidin. Tight junction proteins were visualized by an immunofluorescence method. Rab13, VASP, and ZO-1 were found in apical tight junctions in normal epithelium but were dislocated to the basolateral position in patients with inactive Crohn's disease, whereas the structure of F-actin was maintained in inactive mucosa. In patients with ulcerative colitis, these tight junction proteins were not dislocated. Latent dislocation of tight junction proteins in the inactive mucosa of patients with Crohn's disease may permit the invasion of gut antigens to initiate and perpetuate altered immune response.
\end{abstract}

Correspondence to: Dr Nobuhide Oshitani, Department of Gastroenterology, Osaka City University Graduate School of Medicine 1-4-3 Asahi-machi, Abeno-ku, Osaka 545-8585, Japan

E-mail: gomizmei@med.osaka-cu.ac.jp

Key words: Crohn's disease, vasodilator-stimulated phosphoprotein, Rab13, tight junction

\section{Introduction}

Crohn's disease (CD) and ulcerative colitis (UC) are inflammatory bowel diseases (IBDs), but their etiology is unknown. Epithelial barrier dysfunction, which can be measured as increased intestinal permeability, has been implicated in IBD $(1,2)$, even in quiescent patients (3). Furthermore, $25-41 \%$ of first-degree relatives of CD patients had increased intestinal permeability in the absence of clinical symptoms (4-6). Increased intestinal permeability permits mucosal exposure to luminal stimuli and plays a key role in the initiation of $\mathrm{CD}$. Although increased intestinal permeability has long been implicated in the pathogenesis of $\mathrm{CD}$, the mechanism of the increased permeability is not known.

The intestinal mucosal barrier is established by the single layer of epithelial cells, and the intercellular junctional complexes seal the minute spaces between these cells. The intercellular junctional complexes are composed of tight junctions (TJs), adherens junctions, and desmosomes $(7,8)$. TJs are located at the most apical part of the lateral membrane, and form a continuous belt-like structure that circumferentially wraps around the apical pole of epithelial cells. Freezefracture replica electron microscopy reveals TJs to be a netlike meshwork of strands (9). TJs restrict the movement of potentially harmful materials across the epithelium while allowing diffusion of ions and solutes, and also restrict the movement of lipids and integral membrane proteins between the apical and basolateral domains $(10,11)$. TJs are very important in maintaining intestinal permeability. Abnormalities of TJs may therefore be related to increased intestinal permeability in IBDs.

TJs comprise at least four types of transmembrane proteins, including occludin, claudins, junctional adhesion molecules (JAMs), and Crumb (Crb), as well as scaffolding proteins such as zonula-occludin (ZO-1) and a number of cytoplasmic peripheral proteins such as kinases and GTPases (11). Some studies have reported abnormalities of TJs in IBD. Zeissig et al reported reduced and discontinuous $\mathrm{TJ}$ strands in patients with active Crohn's disease (12). They found up-regulation of 
claudin 2 and down-regulation and redistribution of claudin 5 and 8 in active Crohn's disease (12). We reported dislocation of ZO-1 in inactive Crohn's disease (13). Vasodilator-stimulated phosphoprotein (VASP), one of the TJ cytoplasmic proteins, has actin-binding and actin monomer-nucleating capabilities (14). Thus, VASP could serve as a junctionally associated cytoskeletal link and may have an important role in reestablishment of TJ assembly (15). The small GTPase Rab13 is also involved in regulating TJ dynamics (16-18). Rab13, in its GTP-bound form, interacts with an effector that inhibits the recruitment of claudin 1 and ZO-1 to TJs (19).

Köhler et al $(16,20)$ and Zahraoui (21) reported that Rab13 regulates phosphorylation and recruitment of VASP with protein kinase A (PKA) during TJ assembly. Rab13 and VASP have important roles and correlate in TJ assembly. There have been no reports of the expression of these TJ proteins in patients with IBD. Therefore, the aim of the present study was to analyze the expression of VASP, ZO-1, and Rab13 in the lower intestine in control individuals compared with their expression in the inactive lower intestine of patients with IBD.

\section{Materials and methods}

Patients. Surgical specimens of macroscopically normal or inactive lower intestine were obtained from 10 controls (who had undergone surgery because of intestinal carcinoma: 7 males, 3 females; median age, 75; range, 61-80 years), 10 patients with $\mathrm{CD}$ (who had intestinal stricture and/or were unresponsive to medical treatment; 5 males, 5 females; median age, 30.5; range, 17-38 years) and 7 patients with UC (6 patients were unresponsive to medical therapy, 1 patient had surgery because of colitic cancer; 5 males, 2 females; median age, 55; range, 21-62 years). Patients with CD included 8 patients with ileocolitis and 2 with ileitis. Three patients with CD received infliximab within a month before operation. Eight patients with CD were receiving 5-amino salicylic acid and two patients with $\mathrm{CD}$ were receiving corticosteroids $(>10 \mathrm{mg})$ at the time of surgery. Of the 7 patients with UC, 4 were receiving corticosteroids $(>20 \mathrm{mg})$ at the time of surgery. Duration of disease in patients with $\mathrm{CD}$ ranged from 0.5 to 14 years (median 8 years) and in those with UC ranged from 1 to 22 years (median 3 years). Since intestinal permeability is increased, even in quiescent $\mathrm{CD}$ patients, and TJ abnormalities in inactive mucosa may play a key role in the initiation of $\mathrm{CD}$, we did not investigate the distribution of Rab13 and VASP in active CD and UC.

Immunohistochemistry. Samples were fixed with periodate/ lysine $/ 2 \%$ paraformaldehyde at $4{ }^{\circ} \mathrm{C}$ for $6 \mathrm{~h}$ and then incubated in a $10-15-20 \%$ sucrose gradient in phosphate-buffered saline (PBS) at $4^{\circ} \mathrm{C}$ for $24 \mathrm{~h}$. Specimens were embedded in Tissue-Tek optimum cutting temperature compound (Sakura Fineteck Co., Ltd., Tokyo, Japan) and stored at $-80^{\circ} \mathrm{C}$ until use. Cryostat sections $(4 \mu \mathrm{m})$ were cut from macroscopically inactive mucosa. Sections were pretreated with $5 \%$ non-fat milk for $15 \mathrm{~min}$ to inhibit non-specific antibody binding. After washing, sections were reacted with primary antibodies at $4^{\circ} \mathrm{C}$ overnight. The primary antibodies used were anti-Rab13 goat antibody at a dilution of 1:100, anti-VASP goat antibody (Santa-Cruz Biotechnology, Inc., Santa Cruz, CA, USA) at a dilution of 1:400, anti-phospho-VASP goat antibody at a dilution of a

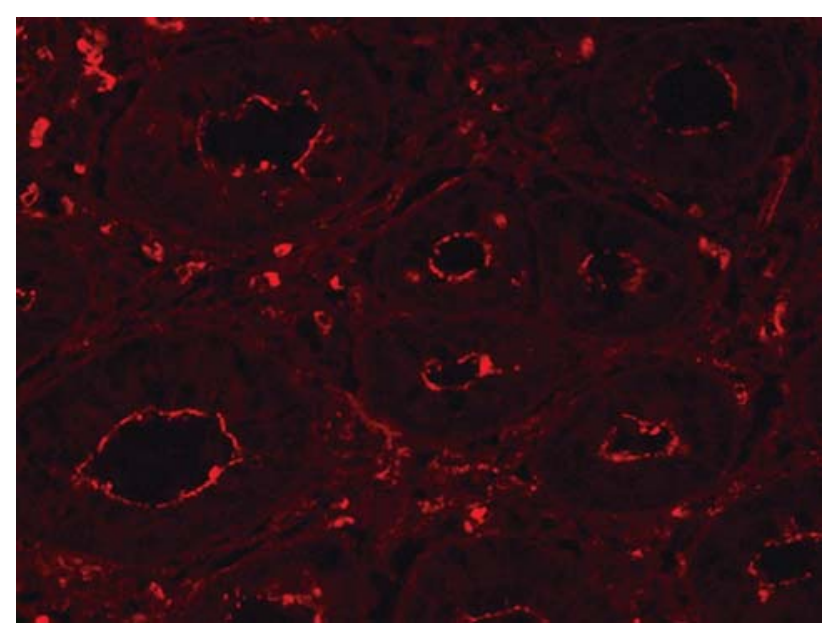

b

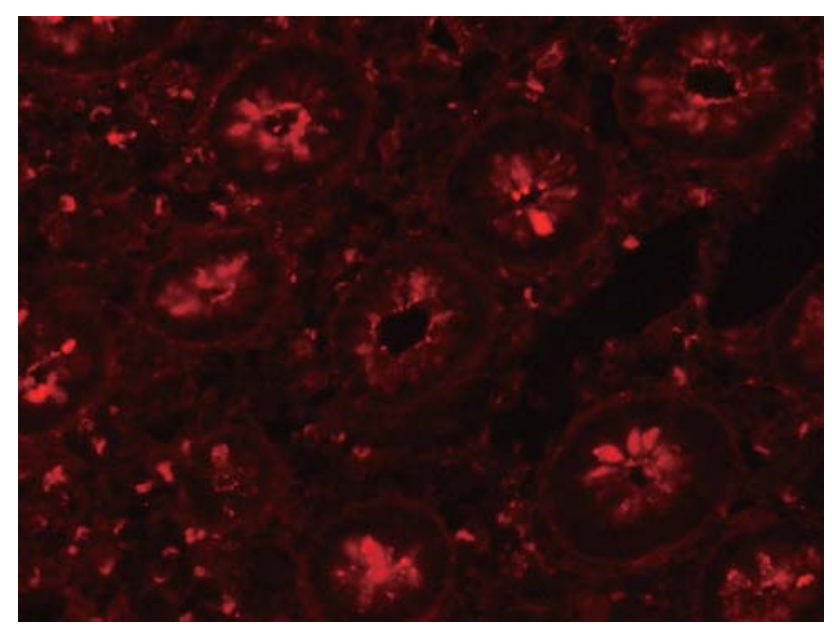

C

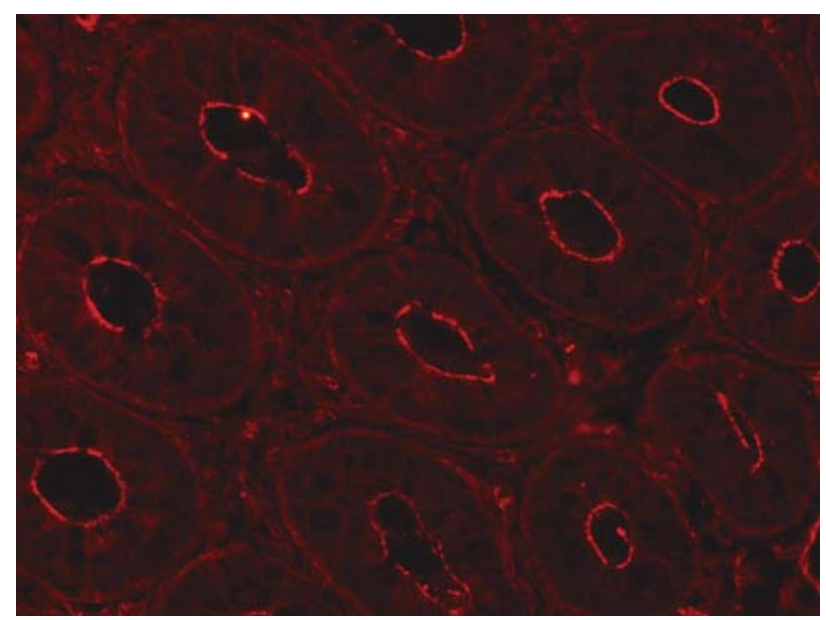

Figure 1. Immunofluorescent visualization of ZO-1 in intestinal mucosa in (a) a control individual, (b) a patient with $\mathrm{CD}$, and (c) a patient with UC. ZO-1 was expressed at the most apical side in the control and the UC patient, whereas in the CD patient $\mathrm{ZO}-1$ was dislocated to the cytoplasm and to mucus in goblet cells

1:100, anti-ZO-1 monoclonal antibody (Invitrogen Corp., Carlsbad, CA, USA) at a dilution of 1:100. After washing three times in PBS containing $0.05 \%$ Tween-20, sections were reacted with secondary antibodies (Alexa Fluor 488 donkey 
a

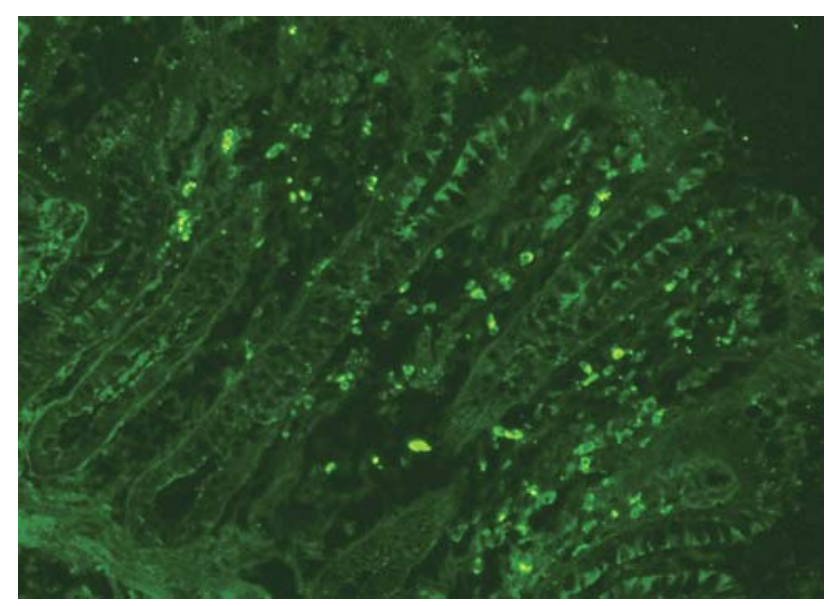

b

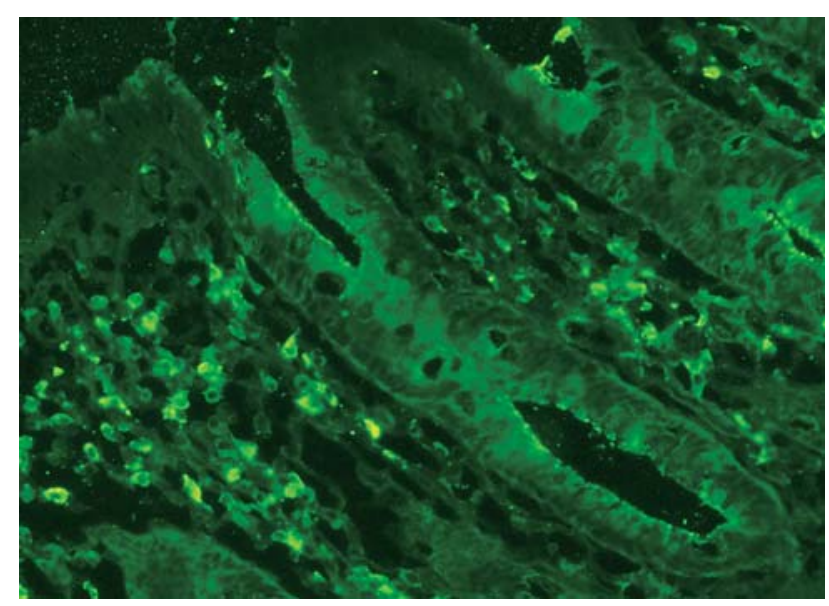

c

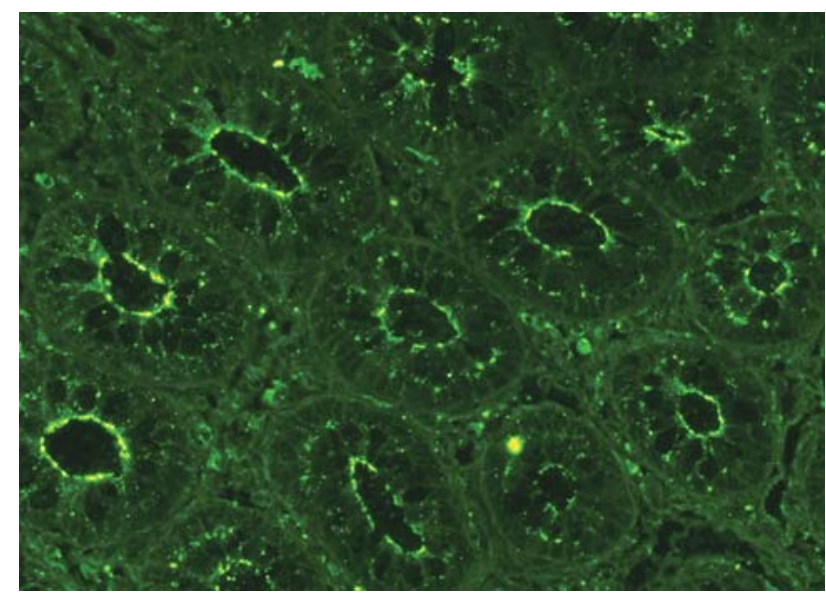

Figure 2. Immunofluorescent visualization of VASP in intestinal mucosa in (a) a control individual, (b) a patient with $\mathrm{CD}$, and (c) a patient with UC. VASP was expressed at the most apical side, similar to the location of ZO-1 in controls and UC patients. In the CD patient, staining of the apical side was weakened, and VASP was dislocated to the cytoplasm.

anti-goat IgG antibody and Alexa Fluor 594 donkey antimouse $\mathrm{IgG}$ antibody (Invitrogen Corp.), both at a dilution of 1:200) at room temperature for $25 \mathrm{~min}$. Alexa Fluor 350 fluorescent phallotoxin (Invitrogen Corp.) was used with secondary antibodies at a dilution of 1:20 to examine the presence of the cytoskeletal protein F-actin. Then, sections were mounted in Fluorescent Mounting Medium (Dakocytomation, Glostrup, Denmark) gel and observed under a fluorescence microscope (Olympus BX50F4, Olympus, Tokyo) equipped with a color camera (Olympus Color Chilled 3CCD camera, M-3204c).

Informed consent was obtained from all patients at the assessment before sampling of tissue specimens. The study was conducted in accordance with the guidelines of Osaka City University's Ethics Committee.

Statistical analysis. Chi-square analysis was used for table analysis. P-values $<0.05$ were considered significant.

\section{Results}

Dislocation of ZO-1. ZO-1 was found at the apical side of normal intestinal epithelial cells, corresponding to the position of the TJs in control mucosa (Fig. 1a). F-actin was expressed in the basolateral and apical membrane in normal epithelium. In 7 of $10 \mathrm{CD}$ patients, the expression of ZO-1 was dislocated from the apical $\mathrm{TJ}$ to the basolateral position and was also found in mucus in goblet cells (Fig. 1b), while the cytoskeletal architecture was well preserved. In all UC patients, ZO-1 was found at the apical side, similar to its location in the control individuals (Fig. 1c).

Dislocation of VASP. VASP was also found at the apical side, corresponding to the position of the TJs in control mucosa (Fig. 2a). In 7 of the 10 CD patients, the expression of VASP was dislocated from the apical TJs to the basolateral position (Fig. 2b). In contrast, in all UC patients except one, VASP was expressed in the form of a line, similar to its distribution in controls (Fig. 2c). Phospho-VASP was not detected in any individual.

Dislocation of Rab13. Rab13 was expressed at the apical side and the supranuclear portion in controls (Fig. 3a). Staining of the supranuclear portion was stronger, and expression was in the form of a line. In the seven CD patients, Rab13 was also dislocated to the cytoplasm and the linear pattern of expression that was seen in normal individuals was absent (Fig. 3b). Rab13 was also found in mucus in goblet cells in most of the $\mathrm{CD}$ patients. In the remaining three cases, staining of Rab13 was similar to that in controls. In contrast, in all UC patients, Rab13 was expressed at the apical side and in the supranuclear portion, similar to its expression in control mucosa (Fig. 3c).

Merge. Colocalization of ZO-1 and peripheral TJ proteins (VASP and Rab13) was studied by immunofluorescent double staining. Merged pictures of normal epithelium showed colocalization of ZO-1 and VASP (Fig. 4a), and ZO-1 and Rab13 (Fig. 4b) at the most apical side. In CD patients, ZO-1 and VASP (Fig. 4c), ZO-1 and Rab13 (Fig. 4d) were dislocated from the apical membrane to the cytoplasm and mucus in goblet cells. Merged pictures of UC patients were similar to that of control (Fig. 4e and f).

Statistical analysis. The dislocations of ZO-1, Rab13, and VASP found in inactive mucosa of CD patients were statistically significant by the chi-square test for independence $(\mathrm{P}<0.001)$ (Tables I and II). 
a

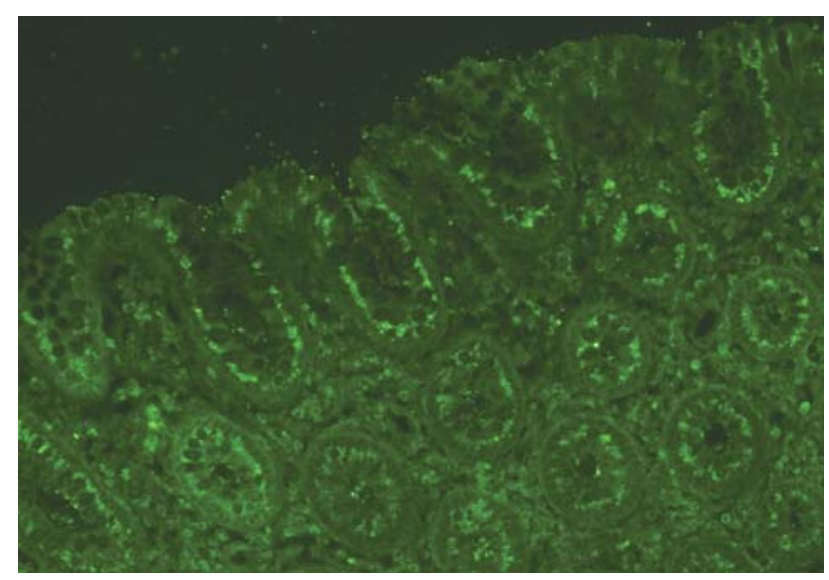

b

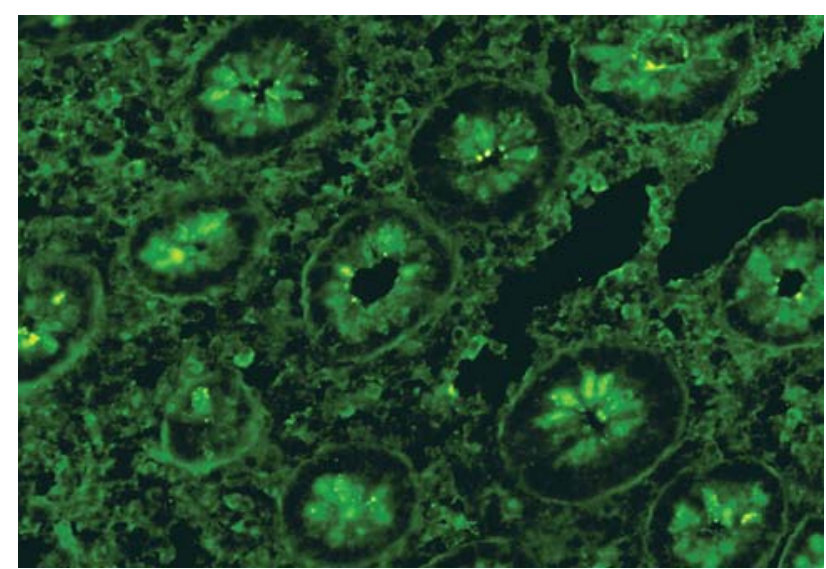

c

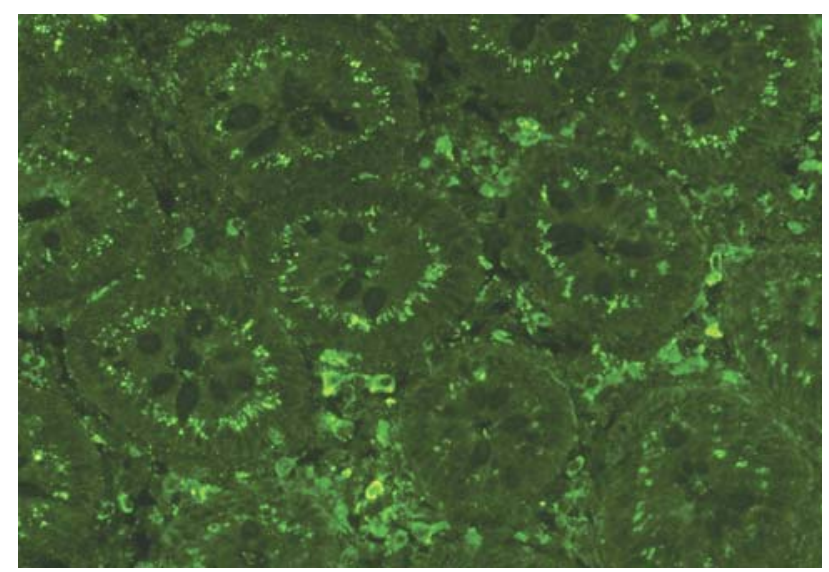

Figure 3. Immunofluorescent visualization of Rab13 in intestinal mucosa in (a) a control individual, (b) a patient with $\mathrm{CD}$, and (c) a patient with UC. Rab13 was expressed at the apical side and in the supranuclear portion in the control (a). Staining of the supranuclear portion was stronger, and the area of expression had a linear shape. In the CD patient, Rab13 was dislocated to the cytoplasm and the linear area of expression that is seen in normal individuals was absent (b). Rab13 was also found in mucus in goblet cells. In contrast, in the UC patient, Rab13 was expressed at the apical side and in the supranuclear portion, similar to its distribution in control mucosa (c).

\section{Discussion}

The results of the present study show that VASP and Rab13 play significant roles in the pathogenesis of $\mathrm{CD}$, but not in
Table I. Dislocation of VASP.

\begin{tabular}{lccr}
\hline & $\begin{array}{c}\text { Dislocation } \\
-\end{array}$ & $\begin{array}{c}\text { Dislocation } \\
+\end{array}$ & Total \\
\hline Control & 10 & 0 & 10 \\
CD & 3 & 7 & 10 \\
UC & 6 & 1 & 7 \\
Total & 19 & 8 & 27 \\
\hline
\end{tabular}

$\mathrm{P}=0.0016$

Table II. Dislocation of Rab13.

\begin{tabular}{lccr}
\hline & $\begin{array}{c}\text { Dislocation } \\
-\end{array}$ & $\begin{array}{c}\text { Dislocation } \\
+\end{array}$ & Total \\
\hline Control & 10 & 0 & 10 \\
CD & 3 & 7 & 10 \\
UC & 7 & 0 & 7 \\
Total & 20 & 7 & 27 \\
\hline
\end{tabular}

$\mathrm{P}=0.0003$

UC. This is the first report describing the dislocation of VASP and Rab13 in CD.

Tight junction (TJ) proteins are important in maintaining intestinal barrier function, which is related to intestinal permeability. Increased intestinal permeability may permit the passage of antigenic molecules leading to active inflammation $(2,22)$. However, the precise mechanisms involved in the regulation of $\mathrm{TJ}$ assembly and the control of intestinal permeability are not fully understood. Several studies have shown increased permeability in a subgroup of patients with inactive $C D$ correlating with a higher relapse rate in these patients $(3,23)$, and also in the asymptomatic relatives of patients with CD (1,4-6). Thus, increased intestinal permeability and $\mathrm{TJ}$ assembly may have a primary role in the pathogenesis of IBD. Here, we investigated the distribution of some TJ proteins in inactive IBD patients.

Tight junction abnormalities in IBD have been reported in several studies. Zeissig et al reported a reduced number of TJ strands, up-regulation of claudin 2, and down-regulation and redistribution of claudin 5 and 8 in active $\mathrm{CD}$ (12). In active UC patients, claudin 2 was also increased. They also reported that the total amount of claudin 1 was not changed, whereas the distribution of claudin 1 was changed in active CD. Also, down-regulation of occludin in active IBD (24) and dislocation of ZO-1 (13) was reported. In addition, expression of junctional adhesion molecule-A (JAM-A) in the intestine is downregulated in active $\mathrm{CD}$ and $\mathrm{UC}$ (25).

The cytoskeletal affiliations of the TJ are believed to play an important role in regulating TJ function $(26,27)$. Because data on F-actin indicate that the structure of the cytoskeleton is not changed in patients with inactive IBD (13), proteins that have an actin-binding capacity are very significant in pathogenesis of IBD. VASP, one of the cytoplasmic peripheral 
a

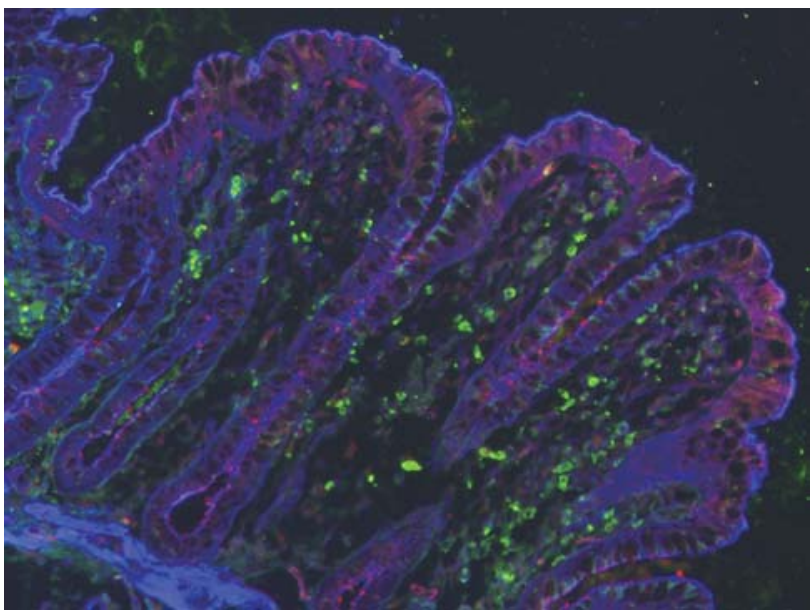

c

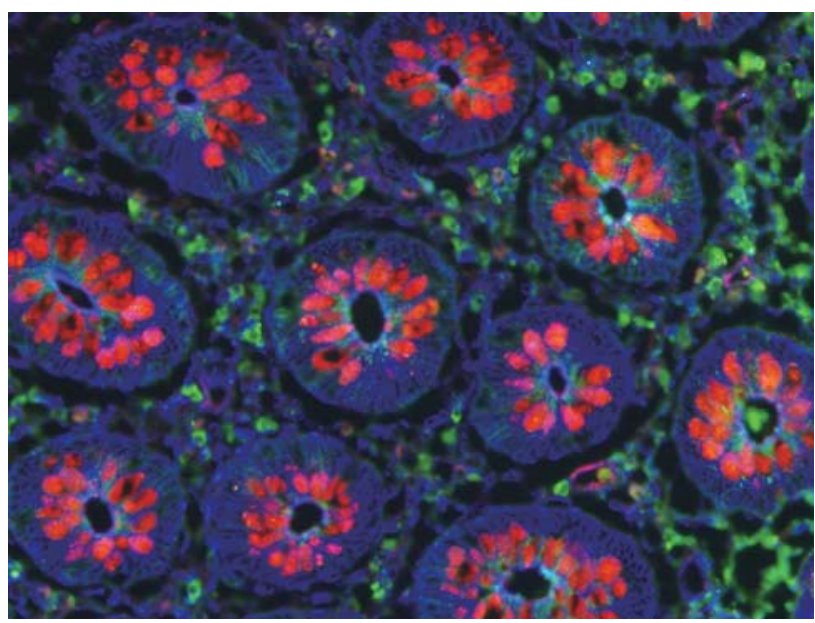

$\mathrm{e}$

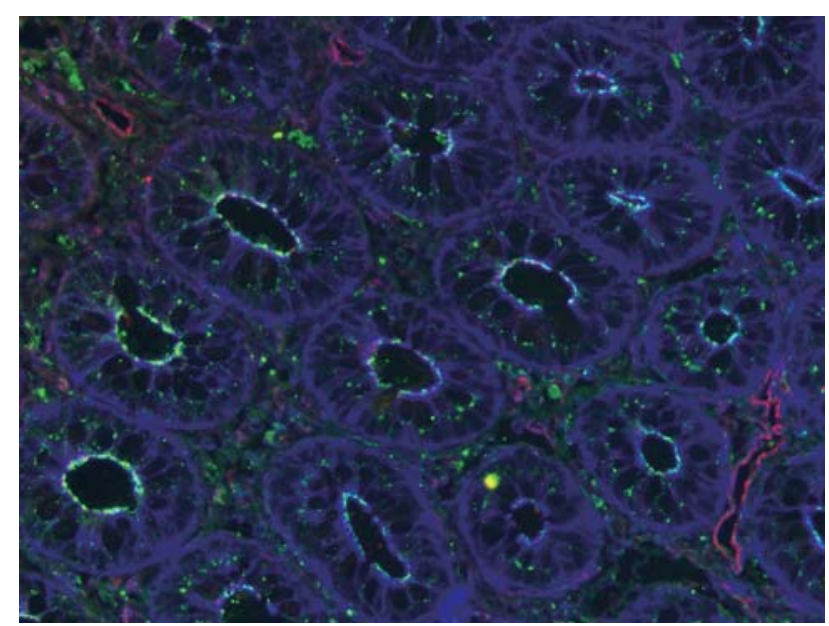

b

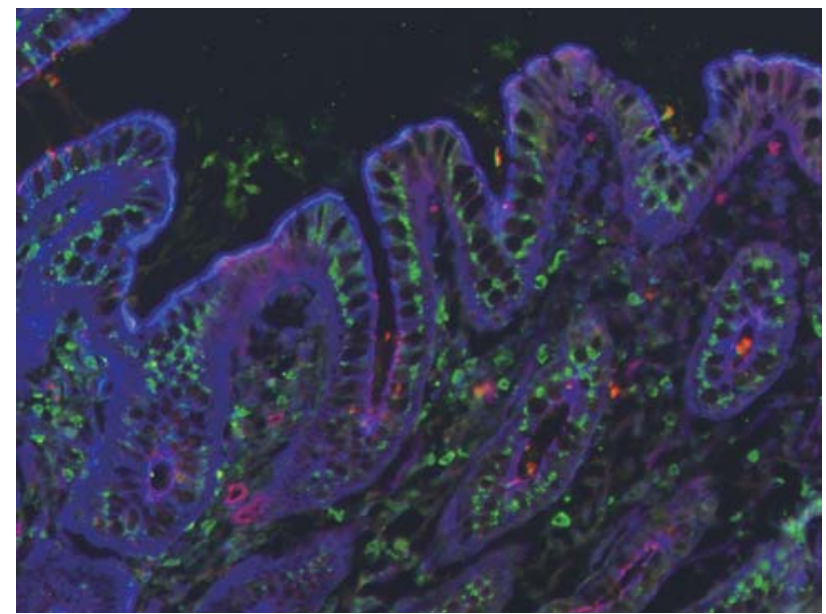

$\mathrm{d}$

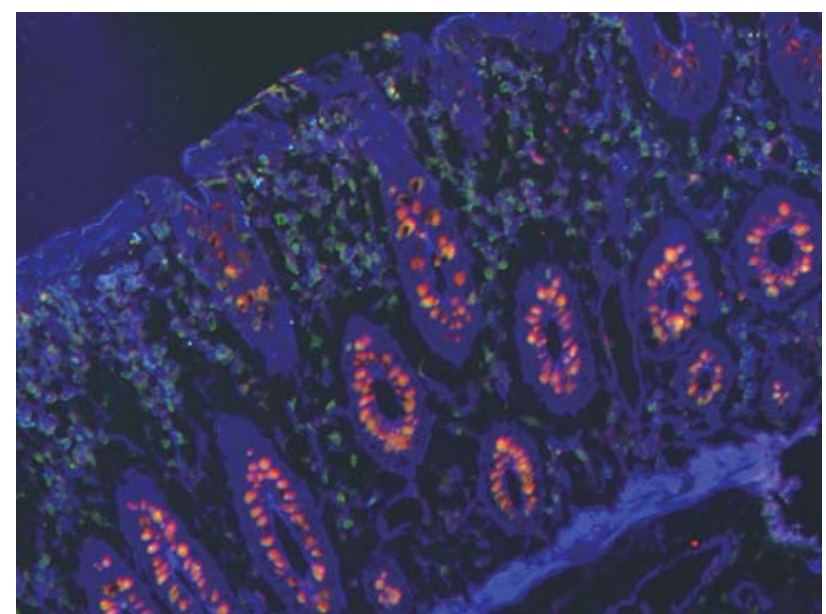

$\mathrm{f}$

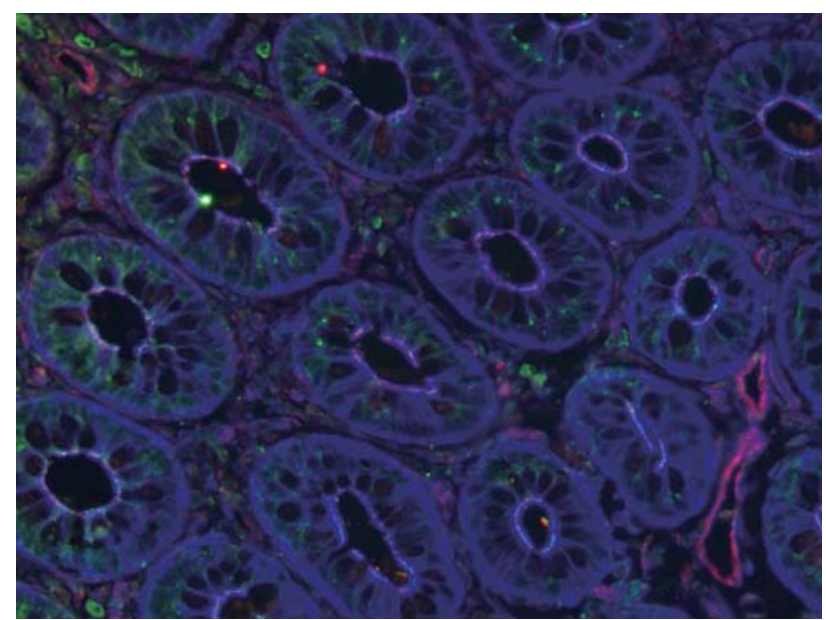

Figure 4. Merged pictures of immunofluorescent staining in intestinal mucosa (a and b) in a control individual (c and d) in a patient with CD, and (e and f) in a patient with UC. In the control, the apical side of the normal epithelium showed white or violet coloring, indicating colocalization of ZO-1 (red), VASP (green), and F-actin (blue) (a), and ZO-1, Rab13 (green), and F-actin (b). In CD patients, the apical membrane was colored blue, indicating only F-actin, and mucus in goblet cells and cytoplasm showed orange coloration, corresponding to colocalization of dislocated ZO-1 and VASP (c), and ZO-1 and Rab13 (d). Merged pictures for the UC patient were similar to those for the control, (e) ZO-1 and VASP; (f) ZO-1 and Rab13.

TJ proteins, was originally discovered as a platelet activation inhibitor (28). Several studies suggested that VASP has an important role in TJ assembly. VASP has actin-binding and cross-linking capacities, and thus could serve as a junctionally associated cytoskeletal link (29). VASP is the regulator of actin cytoskeleton dynamics, and is also involved in actin 
reorganization and directional polymerization. According to Lawrence et al (15) and Harbeck et al (30), ZO-1, a TJ marker, and VASP colocalize at TJs in an epithelial cell model. In our study, VASP and ZO-1 colocalized in the apical site of normal intestinal epithelial cells, corresponding to the position of the TJ. In inactive $\mathrm{CD}, \mathrm{ZO}-1$ and VASP were dislocated from the apical side to the cytoplasm. Interestingly, in inactive UC patients, the locations of ZO-1 and VASP were similar to those in controls. Since VASP is a key actin cytoskeletal remodeling protein, these results suggest that dislocation of ZO-1 would be caused by dislocation of VASP, which is related to the pathogenesis of CD.

VASP has three phosphorylation sites (S157, S239, and T278) shared by PKA and PKG. Phosphorylation at Ser157 by PKA leads to a marked shift in the apparent molecular mass of VASP by SDS-PAGE from 46 to $50 \mathrm{kDa}$ (31). Phospho-VASP (P-VASP) is likely a negative regulator of actin dynamics (30). Lawrence et al suggested that phosphoVASP relaxes cytoskeletal tension, and that this relaxation of the actin cytoskeleton is necessary for junctional assembly (15). Unfortunately, we did not detect P-VASP in the lower intestine by immunohistochemistry. This may be because of technical difficulty or may be related to the method of fixation (32).

Among a number of other cytoplasmic peripheral proteins identified, Rab family small $\mathrm{G}$ proteins are also key regulators of the assembly and maintenance of TJs $(19,33)$. More than 60 Rab family members have been identified in mammalian cells (34). Rab proteins regulate specific membrane transport events, such as vesicle formation, vesicle and organelle motility, tethering, and fusion (33). Rab13 is one of the most important family members for the assembly and maintenance of TJ (35). Yamamura et al, using a $\mathrm{Ca}^{2+}{ }_{-}$switch assay, reported that Rab13 was specifically required for the transport of claudin 1 and occludin from the cytoplasm to the plasma membrane in MDCK cells (19). However, it has not been investigated whether Rab13 controls other claudins. In our study, Rab13 was located at the apical membrane and the supranuclear portion in the epithelium of the normal lower intestine. Rab13 dislocated from these portions to the cytoplasm in patients with inactive $\mathrm{CD}$, but not in patients with inactive UC. Considering the fact that Rab13 is required for the transport of other TJ proteins, Rab13 may also play a key role in the pathogenesis of CD.

According to Zahraoui et al, Rab13 interacts with PKA and reversibly controls the phosphorylation at Ser157 and the recruitment of VASP to cell-cell junctions $(20,21)$. In our study, Rab13 and VASP were both dislocated in inactive CD. These data suggest that Rab13 abnormality may cause the dislocation of VASP and ZO-1 and may be related to the increased intestinal permeability in CD.

In conclusion, we observed that Rab13 and VASP are dislocated in the intestinal epithelium in inactive $\mathrm{CD}$, but not in inactive UC. This suggests that Rab13 and VASP have crucial roles in the pathogenesis of CD.

\section{Acknowledgements}

This work was supported in part by a Grant-in-Aid for Scientific Research of the Japanese Ministry of Education, Culture, Sports, Science and Technology.

\section{References}

1. Hollander D: Crohn's disease - a permeability disorder of the tight junction. Gut 29: 1621-1624, 1988.

2. Bjarnason I: Intestinal permeability. Gut 35: S18-S22, 1994.

3. Arnott IDR, Kingstone $\mathrm{K}$ and Ghosh S: Abnormal intestinal permeability predicts relapse in inactive Crohn disease. Scand J Gastroenterol 35: 1163-1169, 2000.

4. Hilsden RJ, Meddings JB and Sutherland LR: Intestinal permeability changes in response to acetylsalicylic acid in relatives of patients with Crohn's disease. Gastroenterology 110: 1395-1403, 1996.

5. Peeters M, Geypens B, Claus D, et al: Clustering of increased small intestinal permeability in families with Crohn's disease. Gastroenterology 113: 802-807, 1997.

6. Soderholm JD, Olaison G, Lindberg E, et al: Different intestinal permeability patterns in relatives and spouses of patients with Crohn's disease: an inherited defect in mucosal defence? Gut 44: 96-100, 1999.

7. Farquhar MG and Palade GE: Junctional complexes in various epithelia. J Cell Biol 17: 375-412, 1963.

8. Schneeberger EE and Lynch RD: The tight junction: a multifunctional complex. Am J of Physiol Cell Physiol 286: C1213-C1228, 2004.

9. Staehelin LA: Further observations on fine-structure of freezecleaved tight junctions. J Cell Sci 13: 763-786, 1973.

10. Tsukita $\mathrm{S}$ and Furuse $\mathrm{M}$ : Occludin and claudins in tight-junction strands: leading or supporting players? Trends Cell Biol 9: 268-273, 1999

11. Tsukita S, Furuse $M$ and Itoh M: Multifunctional strands in tight junctions. Nat Rev Mol Cell Biol 2: 285-293, 2001.

12. Zeissig S, Burgel N, Gunzel D, et al: Changes in expression and distribution of claudin 2,5 and 8 lead to discontinuous tight junctions and barrier dysfunction in active Crohn's disease. Gut 56: 61-72, 2007.

13. Oshitani N, Watanabe K, Nakamura S, Fujiwara Y, Higuchi K and Arakawa T: Dislocation of tight junction proteins without F-actin disruption in inactive Crohn's disease. Int J Mol Med 15: 407-410, 2005.

14. Reinhard M, Halbrugge M, Scheer U, Wiegand C, Jockusch BM and Walter U: The 46/50-kDa phosphoprotein VASP purified from human platelets is a novel protein associated with actinfilaments and focal contacts. EMBO J 11: 2063-2070, 1992.

15. Lawrence DW, Comerford KM and Colgan SP: Role of VASP in reestablishment of epithelial tight junction assembly after $\mathrm{Ca}^{2+}$ switch. Am J Physiol Cell Physiol 282: C1235-C1245, 2002.

16. Kohler K, Louvard D and Zahraoui A: Rab13 regulates PKA signaling during tight junction assembly. J Cell Biol 165: 175-180, 2004.

17. Marzesco AM, Dunia I, Pandjaitan R, et al: The small GTPase Rab13 regulates assembly of functional tight junctions in epithelial cells. Mol Biol Cell 13: 1819-1831, 2002

18. Marzesco AM and Zahraoui A: Assay of Rab13 in regulating epithelial tight junction assembly. In: GTPases Regulating Membrane Targeting and Fusion. Methods Enzymol 403: 182193, 2005.

19. Yamamura R, Nishimura N, Nakatsuji H, Arase $S$ and Sasaki T: The interaction of JRAB/MICAL-L2 with Rab8 and Rab13 coordinates the assembly of tight junctions and adherens junctions. Mol Biol Cell 19: 971-983, 2008.

20. Kohler K and Zahraoui A: Tight junction: a co-ordinator of cell signalling and membrane trafficking. Biol Cell 97: 659-665, 2005.

21. Zahraoui A: Properties of Rab13 interaction with protein kinase A. In: GTPases Regulating Membrane Targeting and Fusion. Methods Enzymol 403: 723-732, 2005.

22. Mankertz J and Schulzke JD: Altered permeability in inflammatory bowel disease: pathophysiology and clinical implications. Curr Opin Gastroenterol 23: 379-383, 2007.

23. Wyatt J, Vogelsang H, Hubl W, Waldhoer $\mathrm{T}$ and Lochs H: Intestinal permeability and the prediction of relapse in Crohn's disease. Lancet 341: 1437-1439, 1993.

24. Kucharzik T, Walsh SV, Chen J, Parkos CA and Nusrat A: Neutrophil transmigration in inflammatory bowel disease is associated with differential expression of epithelial intercellular junction proteins. Am J Pathol 159: 2001-2009, 2001.

25. Vetrano S, Rescigno M, Cera MR, et al: Unique role of junctional adhesion molecule-A in maintaining mucosal homeostasis in inflammatory bowel disease. Gastroenterology 135: 173-184, 2008. 
26. Madara JL: Intestinal absorptive cell tight junctions are linked to cytoskeleton. Am J Physiol 253: C171-C175, 1987.

27. Madara JL: Regulation of the movement of solutes across tight junctions. Annu Rev Physiol 60: 143-159, 1998.

28. Waldmann R, Nieberding $M$ and Walter U: Vasodilatorstimulated protein-phosphorylation in platelets is mediated by cAMP-dependent and cGMP-dependent protein-kinases. Eur J Biochem 167: 441-448, 1987.

29. Bachmann C, Fischer L, Walter U and Reinhard M: The EVH2 domain of the vasodilator-stimulated phosphoprotein mediates tetramerization, F-actin binding, and actin bundle formation. J Biol Chem 274: 23549-23557, 1999.

30. Harbeck B, Huttelmaier S, Schluter K, Jockusch BM and Illenberger S: Phosphorylation of the vasodilator-stimulated phosphoprotein regulates its interaction with actin. J Biol Chem 275: 30817-30825, 2000.
31. Comerford KM, Lawrence DW, Synnestvedt K, Levi BP and Colgan SP: Role of vasodilator-stimulated phosphoprotein in protein kinase A-induced changes in endothelial junctional permeability. FASEB J 16: 583-585, 2002.

32. Blair SA, Kane SV, Clayburgh DR and Turner JR: Epithelial myosin light chain kinase expression and activity are upregulated in inflammatory bowel disease. Lab Invest 86: 191201, 2006

33. Zerial $\mathrm{M}$ and McBride $\mathrm{H}$ : Rab proteins as membrane organizers. Nat Rev Mol Cell Biol 2: 107-117, 2001.

34. Pfeffer SR: Rab GTPases: specifying and deciphering organelle identity and function. Trends Cell Biol 11: 487-491, 2001.

35. Zahraoui A, Joberty G, Arpin M, et al: A small Rab GTPase is distributed in cytoplasmic vesicles in nonpolarized cells but colocalizes with the tight junction marker ZO-1 in polarized epithelial-cells. J Cell Biol 124: 101-115, 1994. 